\title{
TWO TOPOLOGICAL DEFINITIONS OF A NIELSEN NUMBER FOR COINCIDENCES OF NONCOMPACT MAPS
}

\author{
JAN ANDRES AND MARTIN VÄTH
}

Received 25 August 2003

The Nielsen number is a homotopic invariant and a lower bound for the number of coincidences of a pair of continuous functions. We give two homotopic (topological) definitions of this number in general situations, based on the approaches of Wecken and Nielsen, respectively, and we discuss why these definitions do not coincide and correspond to two completely different approaches to coincidence theory.

\section{Introduction}

The Nielsen number in its original form is a homotopic invariant which provides a lower bound for the number of fixed points of a map under homotopies. Many definitions have been suggested in the literature, and in "topologically good" situations all these definitions turn out to be equivalent.

Having the above property in mind, it might appear most reasonable to define the Nielsen number simply as the minimal number of fixed points of all maps of a given homotopy class. We call this the "Wecken property definition" of the Nielsen number (the reason for this name will soon become clear). However, although this abstract definition has certainly some nice topological aspects, it is almost useless for applications, because there is hardly a chance to calculate this number even in simple situations. Moreover, in most typical infinite-dimensional situations, the homotopy classes are often too large to provide any useful information.

The latter problem is not so severe: instead of considering all homotopies, one could restrict attention only to certain classes of homotopies like compact or so-called condensing homotopies. But the difficulty about the calculation (or at least estimation) of the Nielsen number remains. Therefore, the taken approach is usually different: one divides the fixed point set into several (possibly empty) classes (induced by the map) and proves that certain "essential" classes remain stable under homotopies in the sense that the classes remain nonempty and different. The number of essential classes thus remains stable and this is what is usually called the Nielsen number. In "topologically good"

Copyright (C) 2004 Hindawi Publishing Corporation

Fixed Point Theory and Applications 2004:1 (2004) 49-69

2000 Mathematics Subject Classification: 47H11, 47H09, 47H10, 47H04, 47J05, 54H25

URL: http://dx.doi.org/10.1155/S1687182004308119 
situations, this Nielsen number has the so-called Wecken property, that is, it gives exactly the same number as the above "Wecken property definition" (see, e.g., [12]).

The various approaches to the Nielsen number in literature differ in the way how the classes and "essentiality" are defined. In most approaches, "essentiality" is defined in a homologic way (e.g., with respect to some fixed point index or Lefschetz number). However, in view of the above-described Wecken property definition, and since the existence of a fixed point index or Lefschetz number requires certain additional assumptions on the involved maps, we take in this paper the position that "essentiality" should be defined in a homotopic way instead. The homologic approach (if available) can then be used to prove that a certain class is essential (in the homotopic sense) and in this sense can be used to find lower bounds for the Nielsen number. Such a situation occurs when, for example, one wants to define a Nielsen number for a multivalued condensing map. This was one of our main stimulations of the present paper.

Instead of considering fixed points, one can use essentially the same approach to look also for coincidence points of two maps, intersection points of two maps, or preimage points of a set under a given map. These three aspects were compared with each other and also a homotopic definition of "essentiality" was suggested in [44]. However, it appears that in the infinite-dimensional (i.e., noncompact) situation a different definition is necessary to avoid the problem with too large homotopy classes.

We are mainly interested in a Nielsen number for coincidence points of two continuous maps $p, q: \Gamma \rightarrow X$, that is, in (homotopically stable) lower estimates for the coincidence set

$$
\operatorname{Coin}(p, q):=\{x \in \Gamma: p(x)=q(x)\}
$$

Note that the classical Nielsen number for fixed points is the special case for the situation when $\Gamma=X$ and $p=$ id. If $\Gamma$ and $X$ are both manifolds of the same dimension, the Nielsen number for coincidence points is a classical topic [10, 11, 34, 35, 50] (for more current result, see, e.g., $[13,30,32])$, and it is known that the corresponding number has the Wecken property [36] with some famous exceptions [37]. However, if $\Gamma$ and $X$ have different dimensions or are not even manifolds, the classical theory does not apply (although some approaches are still possible [8]). Nevertheless, one should of course be able to define a Nielsen number in an appropriate way.

There are two different definitions of the Nielsen classes: one is based on the original idea of Nielsen, and the other is based on an idea of Wecken. In the fixed point case ( $p=\mathrm{id}$ ), these definitions turn out to be equivalent. However, in the general setting, these definitions do not coincide and in fact correspond to two different topological approaches to the study of coincidences. We firstly recall these approaches.

\section{The two approaches: epi maps and multivalued theory}

Definition 2.1. Let $X$ be a topological (Hausdorff) vector space, $\Gamma$ a normal space, $\Omega \subseteq$ $\Gamma$ open, and $p, q: \bar{\Omega} \rightarrow X$ continuous. The map $p$ is called $q$-admissible if $\operatorname{Coin}(p, q) \cap$ $\partial \Omega=\varnothing$. 
A $q$-admissible map $p$ is called $q$-epi if, for each continuous map $Q: \bar{\Omega} \rightarrow X$ for which the set $\overline{\operatorname{conv}}((Q-q)(\Omega))$ is compact and which satisfies $Q(x)=q(x)$ on $\partial \Omega$, we have $\operatorname{Coin}(p, Q) \neq \varnothing$.

Clearly, if $p$ is $q$-epi, then $p$ and $q$ have a coincidence point. Moreover, this coincidence point is even homotopically stable, because the property of being $q$-epi is stable under admissible compact perturbations.

Proposition 2.2 (homotopic stability). Let $p$ be q-epi on $\Omega$, and $h:[0,1] \times \bar{\Omega} \rightarrow X$ continuous with $h(0, \cdot)=0$ and compact $\overline{\operatorname{conv}}(h([0,1] \times \Omega))$. Assume in addition that $p-$ $h(t, \cdot)$ is $q$-admissible for each $t \in[0,1]$. Then $p-h(t, \cdot)$ is q-epi for each $t \in[0,1]$.

Proof. It suffices to prove that $p+h(1, \cdot)$ is $q$-epi. Thus, let a map $Q: \bar{\Omega} \rightarrow X$ be given with compact $\overline{\operatorname{conv}}((Q-q)(\Omega))$ and $Q(x)=q(x)$ on $\partial \Omega$. Note that the set

$$
M:=\overline{\operatorname{conv}}((Q-q)(\Omega))+\overline{\operatorname{conv}}(h([0,1] \times \Omega))
$$

is compact and convex. Moreover, by the compactness of $[0,1]$, one can conclude that the canonical projection $\pi:[0,1] \times \bar{\Omega} \rightarrow \bar{\Omega}$ is a closed map. This implies in particular that the set $C:=\bigcup_{t \in[0,1]} \operatorname{Coin}(p-h(t, \cdot), q)$ is closed. Since $C$ is, by the hypothesis, disjoint from $\partial \Omega$, we find by Urysohn's lemma a continuous function $\lambda: \Gamma \rightarrow[0,1]$ with $\left.\lambda\right|_{\partial \Omega}=0$ and $\left.\lambda\right|_{C}=1$. Put $Q_{1}(x):=Q(x)+h(\lambda(x), x)$. Since $M$ is closed and convex, it contains $\overline{\operatorname{conv}}\left(Q_{1}(\Omega)\right)$ which thus is compact. Moreover, for $x \in \partial \Omega$, we have $\lambda(x)=0$, and so $Q_{1}(x)=Q(x)$. Hence, there is some $x_{0} \in \operatorname{Coin}\left(p, Q_{1}\right) \subseteq C$. Since $\lambda\left(x_{0}\right)=1$, it follows that $p\left(x_{0}\right)+h\left(1, x_{0}\right)=Q\left(x_{0}\right)$, that is, $\operatorname{Coin}(p+h(1, \cdot), Q) \neq \varnothing$.

It turns out that if $p^{-1}$ has "sufficiently good" compactness properties, then also certain noncompact homotopies can be considered $[26,56]$.

Proposition 2.3 (restriction property). If $p$ is $q$-epi on $\Omega, \Omega_{0} \subseteq \Omega$ is open, and $\operatorname{Coin}(p, q)$ $\subseteq \Omega_{0}$, then $p$ is q-epi on $\Omega_{0}$.

Proof. Given a continuous $Q: \bar{\Omega}_{0} \rightarrow X$ with compact $\overline{\operatorname{conv}}\left((Q-q)\left(\Omega_{0}\right)\right)$ and $Q(x)=q(x)$ on $\partial \Omega_{0}$, extend $Q$ to $\bar{\Omega}$ by putting $Q(x):=q(x)$ for $x \notin \Omega_{0}$. Since $p$ is $q$-epi, there is some $x_{0} \in \operatorname{Coin}(p, Q)$, and the assumption implies $x_{0} \in \Omega_{0}$.

In the context of Banach spaces and for $q=0$, the corresponding 0-epi maps had been defined for the first time in [22] (see also [31]). The same definition was introduced independently by Granas under the name essential maps (see, e.g., [28]). Meanwhile, the above definition was generalized in many respects; for example, the assumption that $X$ is a (full) vector space could be dropped with some technical effort and also multivalued maps were considered [7]. The crucial property of 0-epi maps is that they are in a sense very similar to maps with nonzero degree: they share the "coincidence point property" $(\operatorname{Coin}(p, q) \neq \varnothing)$, the homotopy invariance (Proposition 2.2), and a weak form of the additivity of the degree (Proposition 2.3). In fact, if a reasonable degree is defined for $p$ : $X \rightarrow X$, then $p$ is 0 -epi if and only if $p$ has nonzero degree [26]. However, it makes sense to speak about $q$-epi maps even if no degree is defined and even in general topological spaces 
(not only in topological vector spaces). In the latter case, one can use the homotopic stability as the definition (see [23]).

Remark 2.4. It will later turn out important that Proposition 2.3 is not a full replacement for the additivity of the degree because its converse is not valid. This somewhat reflects the fact that homotopy theory does not satisfy the excision axiom of homology theory (on which the degree is based).

It appears that besides degree theory, there are no homologic methods available to prove that a map $p: \Gamma \rightarrow X$ is $q$-epi. Currently, we know only about the following homologic methods which might be used to prove that a map is $q$-epi.

(1) If $\Gamma=X$ and $p$ is a compact (or at least condensing) perturbation of the identity, then the Nussbaum-Sadovskil degree might apply (see, e.g., $[1,15,47,49]$ ).

(2) If $\Gamma$ and $X$ are Banach spaces and $p$ is a (compact perturbation of a) linear Fredholm operator with 0 , respectively positive, index, then the Mawhin degree [43] (see also $[25,48]$ ), respectively the Nirenberg degree $[45,46]$, might apply (for an approach which combines this with the multivalued theory described below, see $[24,41])$.

(3) If $\Gamma$ is a Banach space with a dual space $X$ and $p$ is a (compact perturbation of a) uniformly monotone operator, then the Skrypnik degree $[39,53]$ might apply.

At a first glance, it might appear that also the case of a Vietoris map $p$ should belong to this list of homologic methods, because for such maps a powerful coincidence index for pairs $(p, q)$ of maps is known. In fact, this is the known fixed point index of the multivalued map $q p^{-1}$. However, this index is of a different nature, as we will see. In fact, this is the second approach to coincidences which we announced before. For simplicity, we consider only the fixed point degree.

Let in what follows $p: \Gamma \rightarrow X$ be a Vietoris map, that is, $p$ is onto, closed, and proper (i.e., preimages of compact sets are compact; in metric spaces this already implies the closedness), and the fibres $p^{-1}(x)$ are acyclic with respect to the Čech homology with coefficients in the field $\mathbb{Q}$ of rational numbers. In the case of noncompact spaces, we will consider the Čech homology functor with compact carriers (cf. [3] or [27]). If $X$ is "sufficiently nice" (a metric ANR), then one can associate to each open set $\Omega \subseteq X$ and each continuous map $q: p^{-1}(\bar{\Omega}) \rightarrow X$ with relatively compact range a fixed point degree $\operatorname{deg}_{p}(q, \Omega)$ provided that the fixed point set

$$
\begin{aligned}
\operatorname{Fix}(p, q) & =\left\{x: x \in q p^{-1}(x)\right\}=\{p(x): p(x)=q(x)\} \\
& =p(\operatorname{Coin}(p, q))=q(\operatorname{Coin}(p, q))
\end{aligned}
$$

contains no point from $\partial \Omega$. This degree has the following properties.

(1) (Coincidence point property). If $\operatorname{deg}_{p}(q, \Omega) \neq 0$, then $\operatorname{Fix}(p, q) \neq \varnothing$ (which is equivalent to $\operatorname{Coin}(p, q) \neq \varnothing)$.

(2) (Homotopy invariance). If $h:[0,1] \times p^{-1}(\bar{\Omega}) \rightarrow X$ is continuous with precompact range and if $\operatorname{Fix}(p, h(t, \cdot)) \cap \partial \Omega=\varnothing$ for each $t \in[0,1]$, then

$$
\operatorname{deg}_{p}(h(0, \cdot), \Omega)=\operatorname{deg}_{p}(h(0, \cdot), \Omega) .
$$


(3) (Additivity). If $\Omega_{1}, \Omega_{2} \subseteq \Omega$ are disjoint and open in $X$ with $\operatorname{Fix}(p, q) \subseteq \Omega_{1} \cup \Omega_{2}$, then

$$
\operatorname{deg}_{p}(q, \Omega)=\operatorname{deg}_{p}\left(q, \Omega_{1}\right)+\operatorname{deg}_{p}\left(q, \Omega_{2}\right)
$$

The existence of this degree (and a more general index with additional properties) is well known, see, for example, [16, 21, 40, 52, 58] or [27, Sections 50-53]. It can also be generalized for noncompact mappings $[6,55]$. The basic idea for its definition was already employed in [17]: the crucial observation is that, by a theorem of Vietoris, $p$ induces an isomorphism on the corresponding Čech (co)homologies, and using the corresponding inverse, one can proceed analogously to the case when an inverse of $p$ would exist.

We note that the above fixed point index is usually employed to prove the existence of fixed points of multivalued maps $\varphi$. In fact, each upper semicontinuous multivalued map in $X$ with compact acyclic values can be written in the form $\varphi=q p^{-1}$ with a Vietoris map $p$. To see this, let $\Gamma$ be the graph of $\varphi$, and $p$ and $q$ the canonical projections onto the first, respectively second, component. Even a composition of acyclic maps can be written in the form $q p^{-1}$, see [27].

Note that, for the fixed point index, the requirements for $q$ take place on sets of the form $p^{-1}(\Omega)$, where $\Omega$ is an open subset of $X$, while for Definition 2.1, we consider open subsets of $\Gamma$. For this reason, if $p$ is not one-to-one, these two approaches are of a different nature: one should think of the fixed point index as a tool to calculate the fixed points of $q p^{-1}$, while Definition 2.1 is appropriate to calculate the coincidence points (i.e., the fixed points of $\left.p^{-1} q\right)$. Of course, $\operatorname{Fix}(p, q) \neq \varnothing$ if and only if $\operatorname{Coin}(p, q) \neq \varnothing$; however, the cardinality of these sets may differ. Since the Nielsen number is concerned with the cardinality, it is not surprising that the two approaches, if applied to define "essentiality of classes," must differ in their nature.

We note that also for pairs with a nonzero fixed point index, a purely homotopic characterization (in a sense similar to Definition 2.1) can be given [57]. So, despite the first impression about the applied tools, the two approaches cannot be considered as "typical homotopic," respectively "typical homologic." Instead, the authors feel that the first approach, (Definition 2.1) is a "typical homotopic or homologic" approach, while the second approach (by the fixed point index) is of a "typical cohomotopic or cohomologic" nature, but this terminology is of course very vague.

It turns out that for the Nielsen number, the choice of the approach is determined by the definition of coincidence point classes. The first approach corresponds in a sense to the Wecken definition of coincidence point classes, and the second approach corresponds to the definition by Nielsen's original idea. The former definition is based on homotopic paths and the latter on liftings to the universal covering, and so implicitly both definitions refer to the first homotopy group. Unfortunately, this group is nontrivial only if, roughly speaking, the space contains a "hole" of codimension 1. Thus, although all the following theory may sound very general, it can essentially only deal with such a situation (if one is interested in Nielsen numbers larger than 1). However, since in all "good" cases this gives a Nielsen number with the Wecken property, this is the best which can be done. This indicates that actually the Nielsen theory is more involved with the structure of the 
spaces than with the involved maps. This reminds us of the usage of Nielsen theory in Thurston's classification of surfaces (see, e.g., [14] or, for an application, [29]).

\section{Definition by Wecken classes}

The Wecken definition of coincidence point classes has the advantage that it is geometrically easy to understand. The disadvantage is that we will have to impose some restrictions on the space $\Gamma$ which in many cases excludes applications to multivalued maps.

Let $p, q: \Gamma \rightarrow X$ be two continuous maps. We call two points $x_{1}, x_{2} \in \Gamma$ Weckenequivalent if there exists a path joining $x_{1}$ with $x_{2}$ in $\Gamma$ such that the images of this path under $p$, respectively $q$, are homotopic (with fixed endpoints). It is clear that this defines an equivalence relation, and so we can speak of corresponding classes of coincidence points.

Unfortunately, even if $X$ is a "nice" space, $p=\mathrm{id}$, and $\operatorname{Coin}(p, q)$ is compact, it may happen that these classes are not topologically separated, as shown by the following example.

Example 3.1. Let $\Gamma \subseteq R^{2}$ be the topologist's sine curve, that is, the closure of the graph of the function $\sin 1 / x$ on $(0,1], X:=\mathbb{R}^{2}, p(x, y):=(x, y)$, and $q(x, y):=(x, 0)$. Then $\operatorname{Coin}(p, q)=\{0\} \cup\{(1 / n \pi, 0): n=1,2, \ldots\}$ obviously divides into the Wecken classes $\{0\}$ and $\{(1 / n \pi, 0): n=1,2, \ldots\}$.

For this reason, we put the following requirements on our spaces:

(1) $\Gamma$ is a locally pathwise connected normal space;

(2) $X$ is a Hausdorff space and each point in $X$ has a simply connected neighborhood.

Unfortunately, the requirement that $\Gamma$ be locally pathwise connected excludes many applications in the context of multivalued maps, because graphs of (acyclic upper semicontinuous) multivalued maps are typically not locally pathwise connected.

Proposition 3.2. Under the above assumptions, all unions of Wecken classes are closed in $\Gamma$ and relatively open in $\operatorname{Coin}(p, q)$. Moreover, for each Wecken class $C \subseteq \operatorname{Coin}(p, q)$, there is an open set $\Omega \subseteq \Gamma$ with $\Omega \supseteq C=\operatorname{Coin}(p, q) \cap \bar{\Omega}$.

Proof. Let $x_{0} \in \operatorname{Coin}(p, q)$ and let $V \subseteq X$ be a simply connected neighborhood of $p\left(x_{0}\right)=$ $q\left(x_{0}\right)$. There is a pathwise connected neighborhood $U \subseteq \Gamma$ of $x_{0}$ with $p(U) \subseteq V$ and $q(U) \subseteq V$. For any $x \in U \cap \operatorname{Coin}(p, q)$, there is a path from $x_{0}$ to $x$ in $U$ witnessing that $x$ and $x_{0}$ are Wecken-equivalent. Hence, $x_{0}$ is an interior (in Coin $\left.(p, q)\right)$ point of its Wecken class. This proves that the Wecken classes are relatively open.

If $U$ is a union of Wecken classes, then the complement $V:=\operatorname{Coin}(p, q) \backslash U$ is the union of the remaining Wecken classes, and so $U$ and $V$ are both relatively open in $\operatorname{Coin}(p, q)$, and thus also both relatively closed in $\operatorname{Coin}(p, q)$. Since $\operatorname{Coin}(p, q)$ is closed (it is the preimage of the closed diagonal under the continuous map $(p, q))$, it follows that $U$ and $V$ are also closed in $\Gamma$.

Applying this observation on a Wecken class $U:=C$, we find, since $\Gamma$ is normal, an open set $\Omega \subseteq \Gamma$ with $C \subseteq \Omega$ and $\bar{\Omega} \cap V=\varnothing$.

In order to define the notion of an "essential" Wecken class, we must pay attention to the class of homotopies under which our obtained "Nielsen number" is supposed to 
be stable. To make this precise, we assume that a certain family of homotopies is given. Of course, a larger family of homotopies means that our Nielsen number will be "more stable." On the other hand, a larger family will possibly decrease the family of essential classes, that is, it will decrease the Nielsen number.

Since two maps $p$ and $q$ are involved, we will actually not consider homotopies but pairs of homotopies. Thus, let a (nonempty) subset

$$
\mathfrak{H} \subseteq\left\{\left(h_{1}, h_{2}\right) \mid h_{i}:[0,1] \times \Gamma \longrightarrow X \text { continuous }\right\}
$$

be given.

In order to simplify our notation, we require that for each $\left(h_{1}, h_{2}\right) \in \mathfrak{H}$ and each $a, b \in$ $[0,1]$ there is a continuous function $\varphi:[0,1] \rightarrow[\min \{a, b\}, \max \{a, b\}]$ with $\varphi(0)=a$ and $\varphi(1)=b$ such that $\tilde{h}_{i}(t, x):=h_{i}(\varphi(t), x)$ satisfies $\left(\tilde{h}_{1}, \tilde{h}_{2}\right) \in \mathfrak{H}$. (If we would not require this, we would have to require locally the property of Definition 3.3 below.)

By $\mathfrak{P}$ we denote the set of all pairs $(p, q)$ of the form $\left(h_{1}(0, \cdot), h_{2}(0, \cdot)\right)$ with $\left(h_{1}, h_{2}\right) \in \mathfrak{H}$.

Now, we want to define when a Wecken class is called essential. One possible definition is that for all homotopic perturbations of the map, the "corresponding" Wecken class is nonempty. This is the original definition of Brooks $[10,11]$, and we will give a precise formulation later.

However, it is rather technical to make precise what is meant by "corresponding" Wecken class. Therefore, we choose a different definition which is also more natural from the viewpoint of $q$-epi maps: having Definition 2.1 and Proposition 2.2 in mind, it might appear natural to call a class essential if all admissible homotopic perturbations of this class have a coincidence point. Note that the admissibility is crucial for Proposition 2.2, that is, that the homotopies have no coincidence points on the boundary of the considered domain. If a Wecken class is always nonempty, under admissible homotopic perturbations, we call it 1-essential (the precise definition will be given below).

But this straightforward definition alone is not sufficient to prove stability of the corresponding "Nielsen number" (i.e., of the number of 1-essential classes) under nonadmissible homotopies. However, it turns out that it suffices to know that the homotopies are "locally" admissible, if we are allowed to adjust the domain in the course of the homotopy appropriately. Since we can only restrict the domain in Proposition 2.3 and cannot extend it (recall Remark 2.4), the straightforward definition of 1-essential classes is not sufficient for our purpose. So we have to require that our notion of essentiality does not change also under extension of the domain. Unfortunately, this requires a recursive definition: in a sense, we want to define essentiality by the fact that admissible homotopic perturbations are essential. This makes the following definition rather technical.

Maybe this is the reason why we found no similar approach in literature: the only paper with a somewhat related approach is [51] where, however, immediately the existence of an appropriate index was assumed. The latter does not appear natural to us, because, as remarked before, the Nielsen number should be defined in a homotopic way, not by a (homologic) index.

Definition 3.3. Each Wecken class $C \subseteq \Gamma$ of a pair $(p, q) \in \mathfrak{P}$ is called 0 -essential. A Wecken class $C$ is called $n$-essential if the following holds for each $\left(h_{1}, h_{2}\right) \in \mathfrak{H}$ with 
$p=h_{1}(0, \cdot), q=h_{2}(0, \cdot)$ : if there is an open set $\Omega \subseteq \Gamma$ satisfying $\Omega \supseteq C=\bar{\Omega} \cap \operatorname{Coin}(p, q)$ and

$$
\bigcup_{t \in[0,1]} \operatorname{Coin}\left(h_{1}(t, \cdot), h_{2}(t, \cdot)\right) \cap \partial \Omega=\varnothing
$$

and such that the set $D:=\operatorname{Coin}\left(h_{1}(1, \cdot), h_{2}(1, \cdot)\right) \cap \Omega$ is either empty or precisely one Wecken class of the pair $\left(h_{1}(1, \cdot), h_{2}(1, \cdot)\right)$, then $D \neq \varnothing$ and, moreover, $D$ is an $(n-1)$ essential class of $\left(h_{1}(1, \cdot), h_{2}(1, \cdot)\right)$.

If $C$ is $n$-essential for every $n$, then $C$ is called essential.

The (possibly infinite) cardinality $N_{\text {Wecken }}^{\mathfrak{H}}(p, q)$ of the set of essential Wecken classes is called the Nielsen number (with respect to $\mathfrak{H}$ in the Wecken sense).

The crucial property is of course that $N_{\text {Wecken }}^{\mathfrak{H}}(p, q)$ is stable under homotopies which we will prove next.

Note that even if $p$ is a Vietoris map, the corresponding multivalued fixed point index (for pairs) cannot be used to prove that a fixed point class is essential, because one has to verify requirements on subsets $\Omega$ of $\Gamma$ : unfortunately, it does not appear that this fixed point index is valid under restrictions of the maps to subsets of $\Gamma$.

Thus, to our knowledge, the only currently available homologic techniques which allow to prove that a class is essential are the three degree theories mentioned in the first part of the previous section. For the particular choice of the Mawhin degree, one obtains then results in the spirit of $[18,19,20]$; the other degree theories have not been considered yet in this connection.

Theorem 3.4. Suppose, in addition to the above requirements on $\Gamma$ and $X$, that $\Gamma \times[0,1]$ is normal. If $\left(h_{1}, h_{2}\right) \in \mathfrak{H}$ are such that Coin $\left(h_{1}, h_{2}\right)$ is compact, then

$$
N_{\text {Wecken }}^{\mathfrak{H}}\left(h_{1}(0, \cdot), h_{2}(0, \cdot)\right)=N_{\text {Wecken }}^{\mathfrak{H}}\left(h_{1}(1, \cdot), h_{2}(1, \cdot)\right) \text {, }
$$

and these numbers are finite.

The proof of Theorem 3.4 goes along the lines of [51]. We first need some observations concerning the auxiliary pair $(P, Q)$, where $P, Q:[0,1] \times \Gamma \rightarrow X \times[0,1]$ are defined by $P(t, x):=\left(h_{1}(t, x), t\right)$ and $Q(t, x):=\left(h_{2}(t, x), t\right)$. This pair will play the role of "fat homotopies" in the fixed point case (cf. $[38,51])$. For a set $M \subseteq[0,1] \times \Gamma$ and $t \in[0,1]$, we use in the following proof the notation

$$
M_{t}:=\{x:(t, x) \in M\}
$$

Lemma 3.5. For each Wecken class $C$ of $(P, Q)$ and each $t \in[0,1]$, the set $C_{t}$ is either empty or a Wecken class of $\left(h_{1}(t, \cdot), h_{2}(t, \cdot)\right)$. Conversely, all Wecken classes of $\left(h_{1}(t, \cdot), h_{2}(t, \cdot)\right)$ have such a form. 
Proof. The second statement follows from the first one and the fact that Wecken classes are disjoint, because for each point $x \in \operatorname{Coin}\left(h_{1}(t, \cdot), h_{2}(t, \cdot)\right)$, we have trivially that $(x, t) \in$ $\operatorname{Coin}(P, Q)$, and so $x \in C_{t}$, for some Wecken class $C$ of $(P, Q)$.

Suppose that $x_{0} \in C_{t}$ is Wecken-equivalent to $x$ with respect to the pair $\left(h_{1}(t, \cdot)\right.$, $\left.h_{2}(t, \cdot)\right)$, that is, there is some path in $\Gamma$ connecting $x_{0}$ with $x$ witnessing this. Then the canonical embedding of this path into $\Gamma \times\{t\}$ determines that $\left(t, x_{0}\right)$ and $(t, x)$ are Wecken-equivalent with respect to the pair $(P, Q)$, that is, $x \in C_{t}$.

Conversely, suppose that $x_{0}, x \in C_{t}$, that is, that $\left(t, x_{0}\right)$ and $(t, x)$ are Wecken-equivalent with respect to the pair $(P, Q)$, and consider a path $\left(\gamma_{1}, \gamma_{2}\right):[0,1] \rightarrow[0,1] \times \Gamma$ witnessing this, that is, $\gamma_{1}(0)=\gamma_{1}(1)=t, \gamma_{2}(0)=x_{0}, \gamma_{2}(1)=x$, and there is a homotopy $\left(H_{1}, H_{2}\right)$ : $[0,1] \times[0,1] \rightarrow X \times[0,1]$ with fixed endpoints such that $\left(H_{1}, H_{2}\right)(0, \cdot)=P \circ\left(\gamma_{1}, \gamma_{2}\right)$ and $\left(H_{1}, H_{2}\right)(1, \cdot)=Q \circ\left(\gamma_{1}, \gamma_{2}\right)$. In particular, $H_{1}(0, \cdot)=h_{1}\left(t, \gamma_{2}(\cdot)\right)$ and $H_{1}(1, \cdot)=h_{2}\left(t, \gamma_{2}(\cdot)\right)$. Hence, $\gamma_{2}$ and the fixed endpoint homotopy $H_{1}$ determine that $x_{0}$ and $x$ are Weckenequivalent with respect to the pair $\left(h_{1}(t, \cdot), h_{2}(t, \cdot)\right)$.

LEMma 3.6. Under the additional assumptions of Theorem 3.4, the following holds: for each Wecken class $C$ of $(P, Q)$ and each $t_{0} \in[0,1]$, there is a neighborhood of $t_{0}$ such that for each $t$ in this neighborhood, the set $C_{t}$ is an essential Wecken class of $\left(h_{1}(t, \cdot), h_{2}(t, \cdot)\right)$ if and only if $C_{t_{0}}$ is an essential Wecken class of $\left(h_{1}\left(t_{0}, \cdot\right), h_{2}\left(t_{0}, \cdot\right)\right)$.

Proof. By Proposition 3.2, there is some open $\Omega \subseteq \Gamma \times[0,1]$ with $\Omega \supseteq C=\operatorname{Coin}(P, Q) \cap \bar{\Omega}$.

Note that $\operatorname{Coin}(P, Q)=\operatorname{Coin}\left(h_{1}, h_{2}\right)$ is compact by hypothesis. Each point $(x, t) \in C$ has a neighborhood of the form $O \times J$ with some open $O \subseteq \Gamma$ and an open $J \subseteq[0,1]$ such that $O \times J \subseteq \Omega$ and such that $t_{0} \notin \partial J$ (the boundary is understood relative to $[0,1]$ ). By compactness, $C$ is covered by finitely many such neighborhoods. Let $O$ denote the union of such a finite cover. By construction, there is some neighborhood $T$ of $t_{0}$ such that for each $t \in T_{0}$, we have $O_{t}=O_{t_{0}}=: \widetilde{\Omega}$. We may assume that $T=[a, b]$.

If $\widetilde{\Omega}=\varnothing$, we have $C_{t}=C_{t_{0}}=\varnothing$ for all $t \in T$, and so neither $C_{t}$ nor $C_{t_{0}}$ can be an essential Wecken class. Thus, assume that $\widetilde{\Omega} \neq \varnothing$.

Since $C \subseteq O \subseteq \Omega$, it is clear that $\operatorname{Coin}\left(h_{1}(t, \cdot), h_{2}(t, \cdot)\right) \cap \partial \widetilde{\Omega} \neq \varnothing$ for each $t \in T$. We choose some continuous $\varphi:[0,1] \rightarrow T$ with $\varphi(0)=t_{0}$ and $\varphi(1)=t$ such that for $\tilde{h}_{i}(t, x):=$ $h_{i}(\varphi(t), x)$, we have $\left(\tilde{h}_{1}, \tilde{h}_{2}\right) \in \mathfrak{H}$. Then

$$
\operatorname{Coin}\left(\tilde{h}_{1}(\tau, \cdot), \tilde{h}_{2}(\tau, \cdot)\right) \cap \partial \widetilde{\Omega}=\varnothing
$$

for each $\tau \in T$, and so if $C_{t_{0}}$ is $n$-essential for $\left(h_{1}\left(t_{0}, \cdot\right), h_{2}\left(t_{0}, \cdot\right)\right)=\left(\tilde{h}_{1}(0, \cdot), \tilde{h}_{2}(0, \cdot)\right)$, it follows from Definition 3.3 that $\widetilde{\Omega}$ contains a point of an $(n-1)$-essential class of the pair $\left(\tilde{h}_{1}(1, \cdot), \tilde{h}_{2}(1, \cdot)\right)=\left(h_{1}(t, \cdot), h_{2}(t, \cdot)\right)$. Since the only coincidence points of this pair in $\widetilde{\Omega}$ are those from $C_{t}$, it follows that $C_{t}$ is $(n-1)$-essential. In particular, if $C_{t_{0}}$ is essential, then also $C_{t}$ must be essential. Conversely, if $C_{t}$ is $(n)$-essential, then an analogous argument (with $\varphi(0)=t$ and $\left.\varphi(1)=t_{0}\right)$ shows that $C_{t_{0}}$ is $(n-1)$-essential.

Proof of Theorem 3.4. The compactness of $\operatorname{Coin}(P, Q)=\operatorname{Coin}\left(h_{1}, h_{2}\right)$ implies in view of Proposition 3.2 that $(P, Q)$ has only a finite number $N$ of Wecken classes. Lemma 3.5 thus implies that the number of Wecken classes of $\operatorname{Coin}\left(h_{1}(t, \cdot), h_{2}(t, \cdot)\right)$ is at most $N$. Since $(P, Q)$ has at most $N$ Wecken classes, the number $\varepsilon>0$ in Lemma 3.6 can be chosen 
independent of the Wecken class $C$. Lemma 3.6 thus shows that the number of essential Wecken classes of $\left(h_{1}(t, \cdot), h_{2}(t, \cdot)\right)$ of the form $C_{t}$ with a Wecken class $C$ of $(P, Q)$ is locally constant with respect to $t$. By Lemma 3.5 , this means that $N_{\text {Wecken }}^{\mathfrak{H}}\left(h_{1}(t, \cdot), h_{2}(t, \cdot)\right)$ is locally constant with respect to $t$. Since $[0,1]$ is connected, the claim follows.

Before Definition 3.3, we have remarked that Brooks' definition of essentiality (and thus of a Nielsen number) is slightly different. We briefly sketch how Brooks' definition reads in our framework.

Definition 3.7. Let $\hat{C} \subseteq \Gamma$ be a Wecken class of a pair $(p, q) \in \mathfrak{P}$. Given some $\left(h_{1}, h_{2}\right) \in \mathfrak{H}$ with $\left(h_{1}(0, \cdot), h_{2}(0, \cdot)\right)=(p, q)$, let $(P, Q)$ be the corresponding fat homotopy as defined above. By Lemma 3.5, there is precisely one Wecken class $C$ of $(P, Q)$ with $C_{t}=\hat{C}$ for $t=0$.

The class $\hat{C}$ is called Brooks-essential for $\left(h_{1}, h_{2}\right)$ if $C_{t} \neq \varnothing$ for each $t \in[0,1]$. If $\hat{C}$ is Brooks-essential for each $\left(h_{1}, h_{2}\right) \in \mathfrak{H}$ with $\left(h_{1}(0, \cdot), h_{2}(0, \cdot)\right)=(p, q)$, then $\hat{C}$ is called Brooks-essential for $(p, q)$.

The (possibly infinite) cardinality $N_{\text {Brooks }}^{\mathfrak{H}}(p, q)$ of Brooks-essential classes of $(p, q)$ is called the Nielsen number for $\mathfrak{H}$ in Brooks' sense.

The definition is made in such a way that Lemma 3.6 holds (without any additional assumptions), when we replace "essential" by "Brooks-essential." Therefore, the invariance under homotopic perturbations from $\mathfrak{H}$ follows analogously as before.

TheOREM 3.8. The symbol $N_{\text {Brooks }}^{\mathfrak{H}}(p, q)$ is a lower bound for the number of coincidence points of $(p, q)$. Moreover, for each $\left(h_{1}, h_{2}\right) \in \mathfrak{H}$,

$$
N_{\text {Brooks }}^{\mathfrak{H}}\left(h_{1}(0, \cdot), h_{2}(0, \cdot)\right)=N_{\text {Brooks }}^{\mathfrak{H}}\left(h_{1}(1, \cdot), h_{2}(1, \cdot)\right) \text {. }
$$

The following connection with Definition 3.3 is an immediate consequence of Lemma 3.6 and the fact that essential classes are nonempty.

Theorem 3.9. Suppose (in addition to our general requirements) that $\Gamma \times[0,1]$ is normal.

(1) Let $\left(h_{1}, h_{2}\right) \in \mathfrak{H}$ be such that $\operatorname{Coin}\left(h_{1}, h_{2}\right)$ is compact. If a Wecken class of $\left(h_{1}(0, \cdot)\right.$, $\left.h_{2}(0, \cdot)\right)$ is essential, then this class is Brooks-essential for $\left(h_{1}, h_{2}\right)$.

(2) Suppose that Coin $\left(h_{1}, h_{2}\right)$ is compact for every $\left(h_{1}, h_{2}\right) \in \mathfrak{H}$. If a Wecken class of some pair $(p, q) \in \mathfrak{P}$ is essential, then this class is Brooks-essential. In particular,

$$
N_{\text {Brooks }}^{\mathfrak{H}}(p, q) \geq N_{\text {Wecken }}^{\mathfrak{H}}(p, q) .
$$

We note that the assumption that $\operatorname{Coin}\left(h_{1}, h_{2}\right)$ is compact, for every $\left(h_{1}, h_{2}\right) \in \mathfrak{H}$, can simply be achieved by restricting the family $\mathfrak{H}$ correspondingly.

We close this section with a very simple example, where we can estimate the Nielsen number, but where a usual index theory does not apply, because we have a map from one Banach space into another. However, the (homologic) Skrypnik degree does apply and can be used to verify the essentiality of the classes.

Theorem 3.10. Let $1<p<\infty, 1 / p+1 / p^{\prime}=1$, and $H:[0,1] \times \ell_{p} \rightarrow \ell_{p^{\prime}}$ be locally bounded with continuous component functions $H_{n}:[0,1] \times \ell_{p} \rightarrow \mathbb{R}\left(\right.$ i.e., $\left.H=\left(H_{n}\right)_{n}\right)$. Suppose that 
there is some $x_{0} \in \mathbb{R}^{2}$ with $\left(H_{1}(t, x), H_{2}(t, x)\right) \neq x_{0}$, for all $(t, x) \in[0,1] \times \ell_{p}$. Assume that, for each $x \in \ell_{p}$ and each sequence $\left(t_{n}, x_{n}\right) \in[0,1] \times \ell_{p}$, the implication

$$
\left(x_{n}-x, \limsup _{n \rightarrow \infty}\left\langle H\left(t_{n}, x_{n}\right), x_{n}-x\right\rangle \leq 0\right) \Longrightarrow\left\|x_{n}-x\right\| \longrightarrow 0
$$

holds, where $\langle\cdot, \cdot\rangle: \ell_{p^{\prime}} \times \ell_{p} \rightarrow \mathbb{R}$ denotes the canonical pairing. Suppose that $H(0, \cdot)$ has precisely two zeroes $z_{1}, z_{2} \in \ell_{p}$ and that $H\left(0, \cdot-z_{k}\right)$ is odd in a neighborhood of 0 , for $k=$ 1,2 . Assume also that the set $K$ of all $x \in \ell_{p}$ with $0 \in H([0,1], x)$ is bounded.

Finally, assume that there is a continuous path $\gamma$ from $z_{1}$ to $z_{2}$ such that

$$
P(t):=\left(H_{1}(0, \gamma(t)), H_{2}(0, \gamma(t))\right) \in \mathbb{R}^{2}
$$

has nonzero winding number around $x_{0} \in \mathbb{R}^{2}$.

Then $H(1, \cdot)$ has at least two zeroes.

Condition (3.8) is satisfied if $H$ is a compact perturbation of a uniformly monotone operator. In particular, if $\ell_{p}=\ell_{p^{\prime}}$ (i.e., if $p=2$ ), condition (3.8) holds if $H$ is a compact perturbation of the identity. Actually, our proof shows that condition (3.8) can even be slightly relaxed: the full strength of (3.8) is only needed if $t_{n} \equiv 0$; if $t_{n} \equiv t \neq 0$, it suffices that (3.8) holds for those sequences which additionally satisfy $H\left(t_{n}, x_{n}\right) \rightarrow 0$; for all other sequences $\left(t_{n}, x_{n}\right)$, one may replace (3.8) by the milder requirement

$$
\left(x_{n} \rightarrow x, H\left(t_{n}, x_{n}\right) \rightarrow 0,\left\langle H\left(t_{n}, x_{n}\right), x_{n}\right\rangle \longrightarrow 0\right) \Longrightarrow\left\|x_{n}-x\right\| \longrightarrow 0 .
$$

Proof. Our first assumption is equivalent to the demicontinuity of $H$ (i.e., $\left(t_{n}, x_{n}\right) \rightarrow(t, x)$ implies $\left.H\left(t_{n}, x_{n}\right) \rightarrow H(t, x)\right)$. Since the considered spaces are reflexive and separable, condition (3.8) thus implies that if $\Omega \subseteq \Gamma:=\ell_{p}$ is open and bounded, and $0 \notin H([0,1] \times \partial \Omega)$, then the Skrypnik degree $\operatorname{deg}(H(t, \cdot), \Omega, 0)$ is defined and independent of $t$, see [53, Theorem 1.3.1].

We interpret $H$ as a mapping from $[0,1] \times \Gamma$ into $X:=\left(\mathbb{R}^{2} \backslash\left\{x_{0}\right\}\right) \times \ell_{p^{\prime}}$. Then $K=$ Coin $(H, 0)$ is compact. Let $P_{2}: X \rightarrow \mathbb{R}^{2}$ denote the projection onto the first two components. Since the path $P=P_{2} \circ H \circ \gamma$ is not contractible, also $H \circ \gamma$ is not contractible, and so $z_{1}$ and $z_{2}$ belong to two different Wecken classes of the pair $(H(0, \cdot), 0)$. We claim that both these classes are essential with respect to $\mathfrak{H}$, where $\mathfrak{H}$ denotes the family of all pairs $H(\varphi(\cdot), 0)$ with continuous $\varphi:[0,1] \rightarrow[0,1]$ (it follows then that $N_{\text {Wecken }}^{\mathfrak{H}}(H(0, \cdot), 0)=2$ ).

Since $K$ is bounded, $X$ is reflexive, and $H$ is demicontinuous, $K$ is weakly sequentially compact, and (3.8) implies that $K$ is actually compact (and so Theorem 3.4 applies). Since $K$ is bounded, we can by the additivity also define the degree $\operatorname{deg}(H(t, \cdot), \Omega, 0)$ for unbounded open $\Omega \subseteq \ell_{p}$ (namely, as the number $\operatorname{deg}(H(t, \cdot), \Omega \cap B, 0$ ), for a sufficiently large open ball $B$ ).

We prove by induction that whenever a Wecken class $C$ of $(p, q)=\left(H\left(t_{0}, \cdot\right), 0\right) \in \mathfrak{P}$ has the property that for some open $\Omega_{0} \subseteq \Gamma$ with $\Omega_{0} \supseteq C=\bar{\Omega}_{0} \cap \operatorname{Coin}(p, q)$ we have $\operatorname{deg}\left(H\left(t_{0}, \cdot\right), \Omega_{0}, 0\right) \neq 0$, then $C$ is $n$-essential. In fact, let $\Omega \subseteq \Gamma$ be as in Definition 3.3, that is, $\Omega$ is open with $\Omega \supseteq C=\bar{\Omega} \cap \operatorname{Coin}(p, q), 0 \notin H(\varphi([0,1]) \times \partial \Omega)$ for some continuous function $\varphi:[0,1] \times[0,1]$ with $H(\varphi(0), \cdot)=p$, and the set $D$ of all $x \in \Omega$ with 
$H(\varphi(1), x)=0$ is either empty or precisely one Wecken class of the pair $(H(\varphi(1), \cdot), 0)$. Then we have by the homotopy invariance and the additivity of the degree that

$$
\operatorname{deg}(H(\varphi(1), \cdot), \Omega)=\operatorname{deg}(H(\varphi(0), \cdot), \Omega)=\operatorname{deg}\left(H(\varphi(0), \cdot), \Omega_{0}\right) \neq 0,
$$

and so the solution property of the degree implies that $D \neq \varnothing$ and, by the induction assumption, $D$ is an $(n-1)$-essential Wecken class, as required.

It thus suffices to prove that there are sufficiently small neighborhoods $\Omega_{k} \subseteq \Gamma$ ( $k=$ $1,2)$ which contain the Wecken class of $z_{k}$ such that $\operatorname{deg}\left(H(0, \cdot), \Omega_{k}, 0\right) \neq 0$. Since $z_{k}$ are the only zeroes of $H(0, \cdot)$, the corresponding Wecken classes are $\left\{z_{1}\right\}$ and $\left\{z_{2}\right\}$. Since $H\left(0, \cdot-z_{k}\right)$ is odd in each sufficiently small symmetric neighborhood $\Omega_{0}$ about 0 , the Borsuk theorem for the Skrypnik degree [53, Theorem 1.3.5] implies that

$$
\operatorname{deg}\left(H(0, \cdot), \Omega_{0}+z_{k}, 0\right)=\operatorname{deg}\left(H\left(0, \cdot-z_{k}\right), \Omega_{0}, 0\right)
$$

is odd (to see the above equality in our case, apply successively the homotopy invariance with $H\left(0, \cdot-t z_{k}\right)$ and the additivity with $\left.\Omega_{0}+t z_{k}\right)$.

\section{Definition by Nielsen classes}

The Nielsen approach to coincidences of two continuous maps $p: \Gamma \rightarrow X \subseteq Y$ and $q: \Gamma \rightarrow$ $Y$ was essentially elaborated in [4,5] (see also [3] for more detailed proofs). We provide here some details which cannot be found in the above references, but we do not repeat the proofs.

The approach is based on universal coverings $\tilde{X}$ and $\tilde{Y}$ of $X$ and $Y$, respectively (see, e.g., $[9,54])$. We must assume, of course, that such universal coverings exist. This is a consequence of the following assumptions which we make throughout in the sequel:

(1) $X$ and $Y$ are paracompact, connected, and locally contractible;

(2) $p: \Gamma \rightarrow X$ is a Vietoris map;

(3) for each $x \in X$, the restriction of $q$ to the set $p^{-1}(x)$ admits a lift to the universal covering space $\tilde{Y}$, that is, if $p_{Y}: \tilde{Y} \rightarrow Y$ denotes the covering map, then there is a continuous map $\tilde{q}: p^{-1}(x) \rightarrow \tilde{Y}$ with $p_{Y} \circ \tilde{q}=q$ on $p^{-1}(x)$.

The last requirement is automatically satisfied for $x \in X$ if $p^{-1}(x)$ is $\infty$-proximally connected [42]. If $X$ is a metric ANR, the latter is satisfied for the compact set $p^{-1}(x)$ if and only if $p^{-1}(x)$ is a so-called $R_{\delta}$-set, that is, the intersection of a decreasing (countable) sequence of compact contractible spaces (see, e.g., [3]). We point out that, from the viewpoint of applications, the difference between $R_{\delta}$-sets and acyclic sets is not very large. Moreover, the difference between $R_{\delta}$-sets and $\infty$-proximally connected sets can only be seen by some pathologic examples which show that the notions are not equivalent. In a certain sense, if $p^{-1}(x)$ is $\infty$-proximally connected, for each $x$, then $p^{-1}$ is homotopic to a single-valued map. Thus, the above assumption can be interpreted as a homotopic requirement (which is not surprising for the Nielsen number); it is unknown whether the purely homologic condition of acyclicity is sufficient for this.

Besides the universal coverings $p_{X}: \tilde{X} \rightarrow X$ and $p_{Y}: \tilde{Y} \rightarrow Y$, we also need the pullback

$$
\widetilde{\Gamma}:=\left\{(\tilde{x}, z) \in \tilde{X} \times \Gamma: p_{X}(\tilde{x})=p(z)\right\}
$$


together with the canonical projections $\tilde{p}: \widetilde{\Gamma} \rightarrow \tilde{X}$ and $p_{\Gamma}: \widetilde{\Gamma} \rightarrow \Gamma$. It can be proved under our assumptions (see $[3,4,5]$ ) that there is a lift $\tilde{q}$ of $q$ which makes the following diagram commutative:

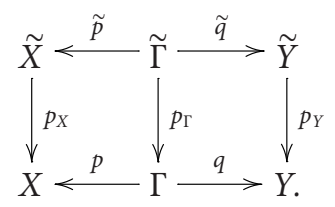

Another ingredient is needed, namely, the deck transformation group

$$
\theta_{X}:=\left\{\alpha: \tilde{X} \longrightarrow \tilde{X} \mid \alpha \text { is a homeomorphism, } p_{X} \alpha=p_{X}\right\} .
$$

The group $\theta_{Y}$ is defined analogously, and

$$
\theta_{\Gamma}:=\left\{(\tilde{x}, z) \in \widetilde{\Gamma} \longmapsto(\alpha \tilde{x}, z): \alpha \in \theta_{X}\right\} .
$$

The lift $\tilde{p}$ in diagram (4.2) defines a group homomorphism $\tilde{p}^{!}: \theta_{X} \rightarrow \theta_{\Gamma}$ by

$$
\tilde{p}^{!}(\alpha)(\tilde{x}, z):=(\alpha(\tilde{x}), z) .
$$

Then the diagram

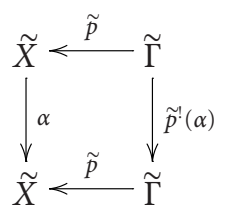

is commutative. Moreover, also $\tilde{q}$ induces a group homomorphism $\tilde{q}_{!}: \theta_{\Gamma} \rightarrow \theta_{Y}$ by the requirement that the diagram

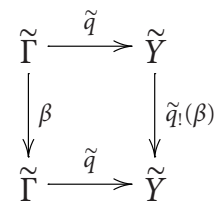

commutes. In particular, (4.2) induces a map $\tilde{q}_{!} \tilde{p}^{!}: \theta_{X} \rightarrow \theta_{Y}$ such that the diagram

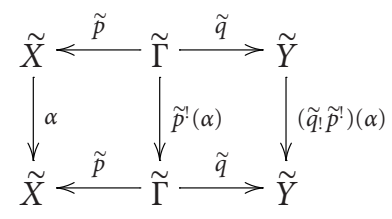

commutes. We point out that $\tilde{q}_{!}$, and thus the composition $\tilde{q}_{!} \tilde{p}^{!}$in general, depend on the choice of the lift $\tilde{q}$. In addition to our previous requirements (1), (2), and (3), we assume now the following: 
(4) $X=Y$;

(5) we fix a normal subgroup $H \subseteq \theta_{X}$ which is invariant under the homomorphism $\tilde{q} ! \tilde{p}^{!}$.

The action of $H$ on $\tilde{X}$ then gives the quotient space $\tilde{X}_{H}$, and the corresponding map $p_{X H}$ induced by $p_{X}$ is also a covering. Moreover, defining the action of $H$ on $\widetilde{\Gamma}$ by $h \circ(\tilde{x}, z):=$ $(h \tilde{x}, z)$, we obtain a quotient space $\widetilde{\Gamma}_{H}$ and corresponding projections $\tilde{p}_{H}$ and $p_{\Gamma H}$ onto the first and the second components. In view of (5), the map $\tilde{q}$ induces a continuous map $\tilde{q}_{H}$, and the diagram

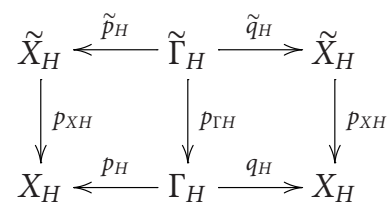

commutes. Since the subgroup $H \subseteq \theta_{X}$ is normal, each $\alpha \in \theta_{X}$ induces a map on $\tilde{X}_{H}$, and thus an element of the set $\theta_{X H}$ of all deck transformations of the covering $p_{X H}$. Conversely, all the elements of $\theta_{X H}$ have such a form, because they are determined by their action on a single point, and $\theta_{X}$ acts transitively on each fibre of the covering. We put

$$
\theta_{\Gamma H}:=\left\{(\tilde{x}, z) \longmapsto(\alpha \tilde{x}, z): \alpha \in \theta_{X H}\right\}
$$

and define $\tilde{p}_{H}^{!}: \theta_{X H} \rightarrow \theta_{\Gamma H}$ by

$$
\tilde{p}_{H}^{!}(\alpha)(\tilde{x}, z):=(\alpha(\tilde{x}), z) .
$$

Finally, we define $\tilde{q}_{H !}: \theta_{\Gamma H} \rightarrow \theta_{X H}$ by the commutativity requirement

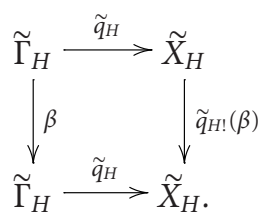

Note that if we keep the covering $p_{X}: \tilde{X} \rightarrow X$ and the subgroup $H$ once and for all fixed, all the above definitions depend besides the pair $(p, q)$ only on the choice of the lift $\tilde{q}$.

Definition 4.1. Two elements $\alpha, \beta \in \theta_{X H}$ are in the $H$-Reidemeister relation with respect to $(p, q, \tilde{q})$ if there is some $\gamma \in \theta_{X H}$ such that

$$
\beta=\gamma \alpha\left(\left(\tilde{q}_{H !} \tilde{p}_{H}^{!}\right)(\gamma)\right)^{-1}
$$

Since the group $\theta_{X H}$ operates onto itself by the right-hand side, it splits into corresponding classes, the $H$-Reidemeister classes.

The following proposition has been proved in $[3,4,5]$ (the last statement only in the case of the trivial group $H$, and the second statement was formulated a bit weaker). 
Proposition 4.2. Given $(p, q)$ and some lift $\tilde{q}$ as above, put

$$
C_{\alpha}:=C_{\alpha}(p, q, \tilde{q}):=p_{\Gamma H}\left(\operatorname{Coin}\left(\tilde{p}_{H}, \alpha \tilde{q}_{H}\right)\right) \quad\left(\alpha \in \theta_{X H}\right)
$$

Then

(1) $\operatorname{Coin}(p, q)$ is the union of the sets $C_{\alpha}$;

(2) the sets $C_{\alpha}$ are pairwise-disjoint and depend (for fixed $(p, q, \tilde{q})$ ) only on the HReidemeister class of $\alpha$;

(3) if $C_{\alpha} \neq \varnothing$, then the set $C_{\alpha}$ determines the $H$-Reidemeister class of $\alpha$ uniquely;

(4) the family $\mathfrak{N}_{H}(p, q):=\left\{C_{\alpha}: \alpha \in \theta_{X H}\right\}$ (but not necessarily $C_{\alpha}$ ) is independent of the choice of the lift $\tilde{q}$ (and thus actually also independent of the choice of the universal covering).

Definition 4.3. Elements of the set $\mathfrak{N}_{H}(p, q)$ above are called the H-Nielsen classes of the pair $(p, q)$.

Remark 4.4. It is important to understand in the following that the particular choice of the space $\Gamma$ in the above definitions plays no role in the following sense: if $\Gamma_{0}$ is another space which is homeomorphic to $\Gamma$ with a homeomorphism $J: \Gamma_{0} \rightarrow \Gamma$, then everything of the above which holds with the pair $(p, q)$ also holds with the pair $(p \circ J, q \circ J)$. In this sense, we "identify" the pair $(p, q)$ with the pair $(p \circ J, q \circ J)$. However, some care has to be taken: we will tacitly understand that the lift of the map $q \circ J$ is then chosen as $\widetilde{(q \circ J)}(x, z):=\tilde{q}(x, J(z))$.

Now we intend to define essential $H$-Nielsen classes in a homotopic way. To this end, let a fixed class $\mathfrak{H}$ of homotopies be given.

We first explain what we mean by a homotopy: since our philosophy now is to formulate assumptions on subsets of $X$, it is not reasonable to require that a homotopy always corresponds to a fixed space $\Gamma$. Rather, we call a pair $(P, Q)$ with continuous maps $P: \Gamma \rightarrow[0,1] \times X$ and $Q: \Gamma \rightarrow Y$ a homotopy if $P$ and $Q$ satisfy our above requirements (1), (2), and (3) (with $[0,1] \times X$ instead of $X$, of course). Such a homotopy induces a family of pairs $\left(P_{t}, Q_{t}\right)$, where $Q_{t}: \Gamma_{t} \rightarrow Y$ is the restriction of $Q$ to $\Gamma_{t}:=P^{-1}(\{t\} \times X)$, and $P_{t}: \Gamma_{t} \rightarrow X$ is defined by $\left(t, P_{t}(x)\right)=P(x)\left(x \in \Gamma_{t}\right)$. We write $\left(P_{t_{0}}, Q_{t_{0}}\right) \sim\left(P_{t_{1}}, Q_{t_{1}}\right)$ for each $t_{0}, t_{1} \in[0,1]$.

This definition becomes natural in connection with Remark 4.4: we tacitly allow to replace $\left(P_{t_{i}}, Q_{t_{i}}\right)(i=0,1)$ by pairs $\left(P_{t_{i}} \circ J_{i}, Q_{t_{i}} \circ J_{i}\right)$, where $J_{i}: \Gamma_{i}^{\prime} \rightarrow \Gamma_{t}$ is a homeomorphism.

By $\mathfrak{P}$ we denote the class of all pairs $\left(P_{t}, Q_{t}\right) \in \mathfrak{P}$ (for all homotopies and all $t \in[0,1]$ ). We assume that each $(p, q) \in \mathfrak{P}$ satisfies our previous requirements (1), (2), and (3) and in addition that assumptions $(2)$ (with $[0,1] \times X)$ and $(3)$ are satisfied for each $(P, Q) \in \mathfrak{H}$. In other words, the requirements (1), (2), and (3) hold for each $(P, Q) \in \mathfrak{H}$.

Each lifting of a homotopy $(P, Q) \in \mathfrak{H}$ is itself a homotopy $(\widetilde{P}, \widetilde{Q})$ (only for $\widetilde{Q}$, there is a choice of the lifting), and to this homotopy there correspond pairs $\left(\widetilde{P}_{t}, \widetilde{Q}_{t}\right)(0 \leq t \leq 1)$ which are liftings of the pairs $\left(P_{t}, Q_{t}\right)$ associated to the original homotopy $(P, Q)$. In this situation, we call for each $t_{1}, t_{2} \in[0,1]$ the pair $\left(\widetilde{Q}_{t_{1}}, \widetilde{Q}_{t_{2}}\right)$ a $(P, Q)$-admissible lifting for the two pairs $\left(P_{t_{1}}, Q_{t_{1}}\right)$ and $\left(P_{t_{2}}, Q_{t_{2}}\right)$. 
Definition 4.5. A pair $\left(\tilde{q}_{0}, \tilde{q}_{1}\right)$ is an $\mathfrak{H}$-admissible lifting for two pairs $\left(p_{0}, q_{0}\right),\left(p_{1}, q_{1}\right) \in \mathfrak{P}$ if it is $(P, Q)$-admissible for some $(P, Q) \in \mathfrak{H}$.

Note that an $\mathfrak{H}$-admissible lifting exists if and only if $\left(p_{0}, q_{0}\right) \sim\left(p_{1}, q_{1}\right)$.

Remark 4.6. If the pairs $\left(p_{0}, q_{0}\right)$ and $\left(p_{1}, q_{1}\right)$ are only identified via two homeomorphisms with pairs $\left(P_{t_{0}}, Q_{t_{0}}\right)$ and $\left(P_{t_{1}}, Q_{t_{1}}\right)$ of a homotopy $(P, Q) \in \mathfrak{H}$ (in the sense of Remark 4.4), then also the notion of $\mathfrak{H}$-admissible liftings has to be understood in the sense of this remark (i.e., the liftings $\left(\tilde{q}_{0}, \tilde{q}_{1}\right)$ have to take the identifying homeomorphisms into account).

Now, we define essential $H$-Nielsen classes in a manner which is somewhat analogous to Definition 3.3.

Definition 4.7. An $H$-Nielsen class $C \in \mathfrak{N}_{H}(p, q)$ is called 0 -essential for a pair $(p, q) \in \mathfrak{P}$ if $C \neq \varnothing$.

For $n=1,2, \ldots$, the class $C$ is called $n$-essential if, for each $\left(p_{1}, q_{1}\right) \in \mathfrak{P}$ with $(p, q) \sim$ $\left(p_{1}, q_{1}\right)$ and for each corresponding $\mathfrak{H}$-admissible lifting $\left(\tilde{q}, \tilde{q}_{1}\right)$, the following holds: there is some $\alpha \in \theta_{X H}$ with $C=C_{\alpha}(p, q, \tilde{q})$ such that $C_{\alpha}\left(p_{1}, q_{1}, \tilde{q}_{1}\right)$ is $(n-1)$-essential for $\left(p_{1}, q_{1}\right)$.

The class $C$ is called essential if $C$ is $n$-essential for each $n$.

The cardinality of the set of $n$-essential $H$-Nielsen classes is denoted by $N_{H}^{\sim,(n)}(p, q)$. The cardinality of the set of essential $H$-Nielsen classes is denoted by $N_{\tilde{H}}^{\sim}(p, q)$.

Before we discuss this definition, we note that it is equivalent to replace "some $\alpha$ " by "every $\alpha$ " in Definition 4.7. More precisely, the class $C_{\alpha}\left(p_{1}, q_{1}, \tilde{q}_{1}\right)$ in the above definition is actually independent of the particular choice of $\alpha$ in the following sense.

Proposition 4.8. If $C$ is $n$-essential and $(p, q) \sim\left(p_{1}, q_{1}\right)$ with a corresponding $\mathfrak{H}$-admissible lifting $\left(\tilde{q}, \tilde{q}_{1}\right)$, then, for every $\alpha, \beta \in \theta_{X H}$ with $C=C_{\alpha}(p, q, \tilde{q})=C_{\beta}(p, q, \tilde{q})$,

$$
C_{\alpha}\left(p_{1}, q_{1}, \tilde{q}_{1}\right)=C_{\beta}\left(p_{1}, q_{1}, \tilde{q}_{1}\right)
$$

and, in case $n \geq 1$, this class is $(n-1)$-essential for $\left(p_{1}, q_{1}\right)$.

We now formulate the main properties of the Nielsen number $N_{\tilde{H}}^{\sim}(p, q)$.

Proposition 4.9. Each n-essential $H$-Nielsen class is $m$-essential for each $m \leq n$. In particular, n-essential H-Nielsen classes are nonempty. Moreover,

$$
N_{H}^{\sim,(0)}(p, q) \geq N_{H}^{\sim,(1)}(p, q) \geq N_{H}^{\sim,(2)}(p, q) \geq \cdots \geq N_{H}^{\sim}(p, q),
$$

and all these numbers are lower bounds for the cardinality of Coin $(p, q)$.

Theorem 4.10 (homotopy invariance). If $\left(p_{0}, q_{0}\right) \sim\left(p_{1}, q_{1}\right)$, then there exists a one-to-one map of the set of 1-essential H-Nielsen classes of $\left(p_{0}, q_{0}\right)$ into the set of 0 -essential H-Nielsen classes of $\left(p_{1}, q_{1}\right)$, and this mapping sends $(n+1)$-essential H-Nielsen classes of $\left(p_{0}, q_{0}\right)$ into $n$-essential $H$-Nielsen classes, and essential classes into essential classes. In particular, $N_{H}^{\sim,(1)}\left(p_{0}, q_{0}\right)$ is a lower bound for the cardinality of Coin $\left(p_{1}, q_{1}\right)$. Moreover,

$$
N_{H}^{\sim,(n)}\left(p_{1}, q_{1}\right) \geq N_{H}^{\sim,(n+1)}\left(p_{0}, q_{0}\right), \quad N_{H}^{\sim}\left(p_{1}, q_{1}\right)=N_{H}^{\sim}\left(p_{0}, q_{0}\right) .
$$


Theorem 4.10 shows impressively that for most applications it suffices to calculate $N_{H}^{\sim,(1)}\left(p_{0}, q_{0}\right)$, that is, for applications it usually suffices to verify that a class is 1-essential (and so actually the complicated recursion in Definition 4.7 is not needed). This is not too surprising. In fact, if we are interested only in a fixed homotopy and let $\mathfrak{H}$ consist only of this homotopy, then each 1-essential class is essential. However, we do not know whether $N_{H}^{\sim,(1)}(p, q)$ is homotopically invariant even if $\mathfrak{H}$ is stable under concatenations of homotopies: Theorem 4.10 only guarantees the homotopy invariance of the possibly smaller number $N_{H}^{\sim}(p, q)$.

Definition 4.7 differs from the homologic definitions in $[3,4,5]$ where an (under additional assumptions defined) Lefschetz number was used to define the notion of essentiality. Of course, if such a Lefschetz number is defined (such that the Lefschetz fixed point theorem holds) and is nonzero, then the corresponding class is nonempty, that is, 0 essential in the sense of Definition 4.7. Moreover, if this Lefschetz number is stable under the homotopies from $\mathfrak{H}$ (which holds of course for the classes of homotopies considered in $[3,4,5])$, then this class is essential in the sense of Definition 4.7. Hence, $N_{H}^{\sim}(p, q)$ is always at least as large as the Nielsen numbers defined in $[3,4,5]$ when $\mathfrak{H}$ corresponds to the class of homotopies considered in these papers. But $N_{H}^{\sim}(p, q)$ might be larger (and is defined for a richer class of maps), so it is - at least from a theoretical point of view-a better homotopically invariant number which estimates the number of coincidences.

Now, we prove the above results. The proof of Proposition 4.9 is rather straightforward.

Proof of Proposition 4.9. Assume that $C \in \mathfrak{N}_{H}(p, q)$ is $n$-essential for some $n \geq 1$. Choose some homotopy $(P, Q) \in \mathfrak{H}$ with $p=P_{t}$ and $q=Q_{t}$ for some $t$. Fix some lift of this homotopy. This lift shows that there is some $\mathfrak{H}$-admissible lifting of the form $(\tilde{q}, \tilde{q})$ for $(p, q)$ and $\left(p_{1}, q_{1}\right):=(p, q)$. Since $C$ is an $H$-Nielsen class, we find some $\alpha \in \theta_{X H}$ with $C=C_{\alpha}(p, q, \tilde{q})$. We have by Definition 4.7 that $C_{\alpha}(p, q, \tilde{q})$ is $(n-1)$-essential for $\left(p_{1}, q_{1}\right)=$ $(p, q)$.

The proof of the other results needs more preparation.

Lemma 4.11. If $\left(\tilde{q}, \tilde{q}_{1}\right)$ is an $\mathfrak{H}$-admissible lifting for $(p, q)$ and $\left(p_{1}, q_{1}\right)$, then $\tilde{q}_{H !} \tilde{p}_{H}^{!}=$ $\tilde{q}_{1 H !} \tilde{p}_{1 H}$. In particular, the H-Reidemeister relation with respect to $(p, q, \tilde{q})$ and $\left(p_{1}, q_{1}, \tilde{q}_{1}\right)$ is the same.

For the trivial group $H$, Lemma 4.11 has been proved in $[3,4,5]$.

Proof of Proposition 4.8. Since $C$ is $n$-essential, it is nonempty by Proposition 4.9. Hence, $C=C_{\alpha}(p, q, \tilde{q})=C_{\beta}(p, q, \tilde{q}) \neq \varnothing$ implies in view of Proposition 4.2 that $\alpha$ and $\beta$ are in $H$-Reidemeister relation with respect to $(p, q, \tilde{q})$. By Lemma $4.11, \alpha$ and $\beta$ are in $H$ Reidemeister relation with respect to $\left(p_{1}, q_{1}, \tilde{q}_{1}\right)$, and so Proposition 4.2 implies (4.15). In case $n \geq 1$, we find by definition some choice of $\alpha$ as above such that $C_{\alpha}\left(p_{1}, q_{1}, \tilde{q}_{1}\right)$ is $(n-1)$-essential for $\left(p_{1}, q_{1}\right)$.

Proof of Theorem 4.10. Fix some $\mathfrak{H}$-admissible lifting $\left(\tilde{q}_{0}, \tilde{q}_{1}\right)$ for $\left(p_{0}, q_{0}\right)$ and $\left(p_{1}, q_{1}\right)$.

Proposition 4.8 implies that for each $(n+1)$-essential Nielsen class $C=C_{\alpha}\left(p_{0}, q_{0}, \tilde{q}_{0}\right)$ to $\left(p_{0}, q_{0}\right)$ there corresponds precisely one $n$-essential Nielsen class $D:=C_{\alpha}\left(p_{1}, q_{1}, \tilde{q}_{1}\right)$ 
of $\left(p_{1}, q_{1}\right)$. Note that this association is actually independent of $n$, that is, the mapping $C \mapsto D$ defined in this way is actually a restriction of the corresponding map for $n=1$. We claim that this mapping (for $n=1$ ) is one-to-one.

Thus, assume that $D$ is, under this map, the image of two 1-essential Nielsen classes $C_{\alpha}:=C_{\alpha}\left(p_{0}, q_{0}, \tilde{q}_{0}\right)$ and $C_{\beta}:=C_{\beta}\left(p_{0}, q_{0}, \tilde{q}_{0}\right)$, that is,

$$
D=C_{\alpha}\left(p_{1}, q_{1}, \tilde{q}_{1}\right)=C_{\beta}\left(p_{1}, q_{1}, \tilde{q}_{1}\right) .
$$

Since $D$ is 0 -essential, that is, $D \neq \varnothing$, Proposition 4.2 implies that $\alpha$ and $\beta$ are in $H$ Reidemeister relation with respect to $\left(p_{1}, q_{1}, \tilde{q}_{1}\right)$. By Lemma 4.11 , we conclude that $\alpha$ and $\beta$ are also in $H$-Reidemeister relation with respect to $\left(p_{0}, q_{0}, \tilde{q}_{0}\right)$, and so Proposition 4.2 implies that actually $C_{\alpha}=C_{\beta}$, as required.

We point out that the map constructed in the proof of Theorem 4.10 is by no means canonical. In fact, even if $\left(p_{0}, q_{0}\right)$ and $\left(p_{1}, q_{1}\right)$ coincide in a canonical way, this map is in general not the identity.

Example 4.12. Let $X:=Y:=S^{1} \subseteq \mathbb{C}, \Gamma:=[0,1] \times S^{1}$, and let $\mathfrak{H}$ contain the pair $(P, Q)$, where $P:=$ id $: \Gamma \rightarrow[0,1] \times X$ and $Q(t, z):=e^{2 \pi i t} z^{3}$. We may identify the restrictions $P_{t}$ and $Q_{t}$ in a canonical way (i.e., via a canonical homeomorphism in the sense of Remark 4.4) for $t=0$ and $t=1$.

Choose $\tilde{X}:=\tilde{Y}:=\mathbb{R}$ with the covering map $t \mapsto e^{2 \pi i t}$, that is, $\theta_{X}=\{t \mapsto t+k: k \in \mathbb{Z}\}$. Put $H:=\{$ id $\}$ and note that

$$
\widetilde{\Gamma}=\left\{\left((t, x),\left(t, e^{i x}\right)\right): t \in[0,1], x \in \mathbb{R}\right\} .
$$

A lifting is $\widetilde{Q}\left((t, x),\left(t, e^{i x}\right)\right):=t+3 x$. Hence, $\left(\widetilde{Q}_{0}, \widetilde{Q}_{1}\right)$ is $\mathfrak{H}$-admissible for $\left(P_{0}, Q_{0}\right)$ and $\left(P_{1}, Q_{1}\right)$, where

$$
\widetilde{Q}_{j}\left((j, x),\left(j, e^{i x}\right)\right):=j+3 x \quad(j=0,1) .
$$

For $\alpha:=$ id, we have $C_{\alpha}\left(P_{0}, Q_{0}, \widetilde{Q}_{0}\right)=\left\{e^{0}\right\}=\{1\}$, but $C_{\alpha}\left(P_{1}, Q_{1}, \widetilde{Q}_{1}\right)=\left\{e^{2 \pi i \cdot(-1 / 2)}\right\}=\{-1\}$.

Some further notes are in order. This second approach described in this section is indeed more involved with the map $q p^{-1}: X \rightarrow X$ (resp., corresponding liftings) as can already be guessed by the fact that a Lefschetz number for such a map was used for the definition in the cited papers. Thus, it is somewhat surprising that the corresponding Nielsen number is a lower bound for the number of coincidences Coin $(p, q)$, but not on the number of fixed points $\operatorname{Fix}(p, q)$ as shown by [5, Example 2.1] (see also [3]). On the other hand, in this example, the map $q p^{-1}$ does not assume simply connected values. So we formulate the following conjecture.

Conjecture 4.13. If, in addition to the above requirements, the multivalued map $q p^{-1}$ assumes only simply connected values, then $N_{H}^{\sim}(p, q)$ is a lower bound for the number of elements in $\operatorname{Fix}(p, q)$.

The conjecture is natural not only from our "philosophical" considerations about the multivalued approach, but also from the fact that there exist several successful attempts 
in the literature to define a Nielsen number which gives a lower bound on the number of fixed points of multivalued maps with "nice" images, namely $[2,33,42]$.

\section{Acknowledgments}

The authors want to thank L. Górniewicz and J. Jezierski for valuable comments and suggestions. The paper was written while the second author was a Heisenberg Fellow of the DFG (Az. VA 206/1-1) and invited by the University of Olomouc. Financial support by the DFG is gratefully acknowledged. The first author is supported by the Council of Czech Government (J/1498: 153100 011).

\section{References}

[1] R. R. Akhmerov, M. I. Kamenskiĭ, A. S. Potapov, A. E. Rodkina, and B. N. Sadovskiŭ, Measures of Noncompactness and Condensing Operators, Operator Theory: Advances and Applications, vol. 55, Birkhäuser Verlag, Basel, 1992.

[2] J. Andres, Multiple bounded solutions of differential inclusions: the Nielsen theory approach, J. Differential Equations 155 (1999), no. 2, 285-310.

[3] J. Andres and L. Górniewicz, Topological Fixed Point Principles for Boundary Value Problems, Topological Fixed Point Theory and Its Applications, vol. 1, Kluwer Academic Publishers, Dordrecht, 2003.

[4] J. Andres, L. Górniewicz, and J. Jezierski, Noncompact version of the multivalued Nielsen theory and its application to differential inclusions, Differential Inclusions and Optimal Control (J. Andres, L. Górniewicz, and P. Nistri, eds.), Lecture Notes in Nonlinear Analysis, vol. 2, Juliusz Schauder Center for Nonlinear Studies, Torun, 1998, pp. 33-50.

[5] _ A generalized Nielsen number and multiplicity results for differential inclusions, Topology Appl. 100 (2000), no. 2-3, 193-209.

[6] J. Andres and M. Väth, Coincidence index for noncompact mappings in nonconvex sets, Nonlinear Funct. Anal. Appl. 7 (2002), no. 4, 619-658.

[7] J. Appell, M. Väth, and A. Vignoli, $\mathscr{F}$-epi maps, Topol. Methods Nonlinear Anal. 18 (2001), no. 2, 373-393.

[8] L. D. Borsari and D. L. Gonçalves, Obstruction theory and minimal number of coincidences for maps from a complex into a manifold, Topol. Methods Nonlinear Anal. 21 (2003), no. 1, $115-130$.

[9] G. E. Bredon, Topology and Geometry, Graduate Texts in Mathematics, vol. 139, SpringerVerlag, New York, 1993.

[10] R. Brooks, Coincidences, roots and fixed points, Ph.D. thesis, University of California, Los Angeles, 1967.

[11] On removing coincidences of two maps when only one, rather than both, of them may be deformed by a homotopy, Pacific J. Math. 40 (1972), 45-52.

[12] R. F. Brown, Wecken properties for manifolds, Nielsen Theory and Dynamical Systems (South Hadley, Mass, 1992) (C. K. McCord, ed.), Contemporary Mathematics, vol. 152, American Mathematical Society, Rhode Island, 1993, pp. 9-21.

[13] R. F. Brown and H. Schirmer, Nielsen coincidence theory and coincidence-producing maps for manifolds with boundary, Topology Appl. 46 (1992), no. 1, 65-79.

[14] A. J. Casson and S. A. Bleiler, Automorphisms of Surfaces after Nielsen and Thurston, London Mathematical Society Student Texts, vol. 9, Cambridge University Press, Cambridge, 1988.

[15] K. Deimling, Nonlinear Functional Analysis, Springer-Verlag, Berlin, 1985.

[16] Z. Dzedzej, Fixed point index theory for a class of nonacyclic multivalued maps, Dissertationes Math. (Rozprawy Mat.) 253 (1985), 1-53. 
[17] S. Eilenberg and D. Montgomery, Fixed point theorems for multi-valued transformations, Amer. J. Math. 68 (1946), 214-222.

[18] M. Fečkan, Nielsen fixed point theory and nonlinear equations, J. Differential Equations 106 (1993), no. 2, 312-331.

[19]_, Differential equations with nonlinear boundary conditions, Proc. Amer. Math. Soc. 121 (1994), no. 1, 103-111.

[20] _ Multiple solutions of nonlinear equations via Nielsen fixed-point theory: a survey, Nonlinear Analysis in Geometry and Topology (T. M. Rassias, ed.), Hadronic Press, Florida, 2000, pp. 77-97.

[21] G. Fournier and D. Violette, A fixed point index for compositions of acyclic multivalued maps in Banach spaces, Operator Equations and Fixed Point Theorems (S. P. Singh, V. M. Sehgal, and J. H. W. Burry, eds.), vol. 1, The MSRI-Korea Publications, Seoul, 1986, pp. 203-224.

[22] M. Furi, M. Martelli, and A. Vignoli, On the solvability of nonlinear operator equations in normed spaces, Ann. Mat. Pura Appl. (4) 124 (1980), 321-343.

[23] M. Furi and A. Vignoli, An extension of the notion of zero-epi maps to the context of topological spaces, Z. Anal. Anwendungen 21 (2002), no. 2, 485-493.

[24] D. Gabor and W. Kryszewski, A coincidence theory involving Fredholm operators of nonnegative index, Topol. Methods Nonlinear Anal. 15 (2000), no. 1, 43-59.

[25] R. E. Gaines and J. L. Mawhin, Coincidence Degree, and Nonlinear Differential Equations, Lecture Notes in Mathematics, vol. 568, Springer-Verlag, Berlin, 1977.

[26] E. Giorgieri and M. Väth, A characterization of 0-epi maps with a degree, J. Funct. Anal. 187 (2001), no. 1, 183-199.

[27] L. Górniewicz, Topological Fixed Point Theory of Multivalued Mappings, Mathematics and Its Applications, vol. 495, Kluwer Academic Publishers, Dordrecht, 1999.

[28] A. Granas, Continuation method for contractive maps, Topol. Methods Nonlinear Anal. 3 (1994), no. 2, 375-379.

[29] J. Guaschi, Nielsen theory, braids and fixed points of surface homeomorphisms, Topology Appl. 117 (2002), no. 2, 199-230.

[30] J. Guo and P. R. Heath, Equivariant coincidence Nielsen numbers, Topology Appl. 128 (2003), no. 2-3, 277-308.

[31] D. H. Hyers, G. Isac, and T. M. Rassias, Topics in Nonlinear Analysis \& Applications, World Scientific Publishing, New Jersey, 1997.

[32] C.-G. Jang and S. Lee, A relative Nielsen number in coincidence theory, J. Korean Math. Soc. 32 (1995), no. 2, 171-181.

[33] J. Jezierski, The Nielsen relation for multivalued maps, Serdica Math. J. 13 (1987), no. 2, 174181.

[34] The coincidence Nielsen number for maps into real projective spaces, Fund. Math. 140 (1992), no. 2, 121-136.

[35] , The Nielsen coincidence theory on topological manifolds, Fund. Math. 143 (1993), no. 2, $167-178$.

[36] - One codimensional Wecken type theorems, Forum Math. 5 (1993), no. 5, 421-439.

[37] $\quad$, The least number of coincidence points on surfaces, J. Austral. Math. Soc. Ser. A 58 (1995), no. 1, 27-38.

[38] B. J. Jiang, Lectures on Nielsen Fixed Point Theory, Contemporary Mathematics, vol. 14, American Mathematical Society, Rhode Island, 1983.

[39] A. P. Kovalenok and P. P. Zabrěko, The Skrypnik degree and boundary value problems, to appear in Topol. Methods Nonlinear Anal.

[40] W. Kryszewski, The fixed-point index for the class of compositions of acyclic set-valued maps on ANR-s, Bull. Sci. Math. 120 (1996), no. 2, 129-151. 
[41] Homotopy Properties of Set-Valued Mappings, Nicolaus Copernicus University Press, Toruń, 1997.

[42] W. Kryszewski and D. Miklaszewski, The Nielsen number of set-valued maps. An approximation approach, Serdica Math. J. 15 (1989), no. 4, 336-344.

[43] J. Mawhin, Equivalence theorems for nonlinear operator equations and coincidence degree theory for some mappings in locally convex topological vector spaces, J. Differential Equations 12 (1972), 610-636.

[44] C. K. McCord, The three faces of Nielsen: coincidences, intersections and preimages, Topology Appl. 103 (2000), no. 2, 155-177.

[45] L. Nirenberg, An application of generalized degree to a class of nonlinear problems, Troisième Colloque sur l'Analyse Fonctionnelle (Liège, 1970), Vander, Louvain, 1971, pp. 57-74.

[46] - Generalized degree and nonlinear problems, Contributions to Nonlinear Functional Analysis (Proc. Sympos., Math. Res. Center, Univ. Wisconsin, Madison, Wis., 1971) (E. H. Zarantonello, ed.), Academic Press, New York, 1971, pp. 1-9.

[47] R. D. Nussbaum, The fixed point index for local condensing maps, Ann. Mat. Pura Appl. (4) 89 (1971), 217-258.

[48] J. Pejsachowicz and A. Vignoli, On the topological coincidence degree for perturbations of Fredholm operators, Boll. Un. Mat. Ital. B (5) 17 (1980), no. 3, 1457-1466.

[49] B. N. Sadovskiü, Limit-compact and condensing operators, Uspehi Mat. Nauk 27 (1972), no. 1(163), 81-146 (Russian), translated in Russian Math. Surveys 27 (1972), no. 1, 85155.

[50] H. Schirmer, Mindestzahlen von Koinzidenzpunkten, J. reine angew. Math. 194 (1955), 21-39 (German).

[51] U. K. Scholz, The Nielsen fixed point theory for noncompact spaces, Rocky Mountain J. Math. 4 (1974), 81-87.

[52] H. W. Siegberg and G. Skordev, Fixed point index and chain approximations, Pacific J. Math. 102 (1982), no. 2, 455-486.

[53] I. V. Skrypnik, Nonlinear Elliptic Boundary Value Problems, Teubner-Texte zur Mathematik, vol. 91, BSB B. G. Teubner Verlagsgesellschaft, Leipzig, 1986.

[54] E. Spanier, Algebraic Topology, McGraw-Hill Book, New York, 1966.

[55] M. Väth, An axiomatic approach to a coincidence index for noncompact function pairs, Topol. Methods Nonlinear Anal. 16 (2000), no. 2, 307-338.

[56] Coincidence points of function pairs based on compactness properties, Glasg. Math. J. 44 (2002), no. 2, 209-230.

[57] Coepi maps and generalizations of the Hopf extension theorem, Topology Appl. 131 (2003), no. 1, 79-99.

[58] D. Violette and G. Fournier, Un indice de point fixe pour les composées de fonctions multivoques acycliques dans des espaces de Banach [A fixed point index for compositions of acyclic multivalued functions in Banach spaces], Ann. Sci. Math. Québec 22 (1998), no. 2, 225-244 (French).

Jan Andres: Department of Mathematical Analysis, Faculty of Science, Palacký University, Tomkova 40, 77900 Olomouc-Hejčín, Czech Republic

E-mail address: andres@risc.upol.cz

Martin Väth: Department of Mathematics, University of Würzburg, Am Hubland, D-97074 Würzburg, Germany

Current address: Fachbereich Mathematik und Informatik (WE1), Freie Universität Berlin, Arnimallee 2-6, 14195 Berlin, Germany

E-mail address: vaeth@mathematik.uni-wuerzburg.de 